\title{
Emerging Research Communities of Practice vs. the Popular Vision of Interdisciplinarity? Insights from Digital Research in the UK
}

\author{
Yupei Zhao ${ }^{1}$ \\ Panayiota Tsatsou ${ }^{2}$
}

\begin{abstract}
Objective. This paper shows how social science and humanities researchers in the UK who make use of digital tools, resources and services understand and perceive interdisciplinarity and their related experiences and needs.

Methods. The study examined ten cases of UK-based research, two from each of the following social science and humanities disciplines: business/management, education, history, literature and politics. Data collection employed a qualitative methodology that consisted of nonparticipant observation and semistructured interviews.

Results. The paper finds that researchers problematize the meaning and top-down character of interdisciplinarity and envisage the development of research communities of experience exchange and knowledge sharing that go beyond the imperative of interdisciplinarity.

Conclusion. The paper challenges prevalent assumptions that digital research and interdisciplinarity go hand-in-hand and one is a prerequisite for and in need of the other, while inviting institutional and funding bodies to consider working jointly with researchers towards developing the alternative of research communities of practice.
\end{abstract}

\section{Keywords}

Communities of practice, digital research, humanities, interdisciplinarity, research communities of practice, qualitative methodology, social science, UK

Acknowledgment. This work was supported by an EPSRC Community and Culture Network + award (RS15G0111).

\footnotetext{
${ }^{1}$ Email: yupei@yng.media; Correspondence address: School of Communication and Design, Sun Yat-sen University, Panyu, Guangzhou, 510006, China.

${ }^{2}$ Email: pt133@le.ac.uk; Correspondence address: School of Media, Communication \& Sociology, University of Leicester, 132 New Walk, Leicester LE1 7JA, UK.
} 


\section{Introduction}

Scholarly work presents interdisciplinarity as a phenomenon of increasing epistemological and methodological interest (Goulden et al., 2016; Hutchinson, 2016; Mills and Ratcliffe, 2012), marked by certain advantages (Klein, 1996; Salter and Hearn, 1996) and complexities (Bammer, 2013, 2015; Dini et al., 2011).

The increasing presence and role of fast-developing information, communication and computing technologies in conducting scientific research have given rise to the spectacular development of digital research over the last two decades and to related arguments concerning the interdisciplinary nature of digital research work and advancements (Dini et al., 2011; Tsatsou, 2014). Literature (Bammer, 2013, 2015; Holmberg and Thelwall, 2014; Pohoryles and Alice, 2011; Tsatsou, 2016) poses questions with regard to what is meant by interdisciplinarity in digital research, why researchers from different disciplines decide to collaborate in this research field, and how they can effectively practice interdisciplinarity.

Such developments, arguments and questions inspired this study to explore the current picture of interdisciplinarity in social science and humanities research in the UK which employs digital tools, resources and services. More specifically, the aim of the study was to offer original insights that add to ongoing debates on the meaning and importance of interdisciplinarity in the field of digital research. It also aimed to unpack and problematise prevalent assumptions that digital research and interdisciplinarity go hand-in-hand, and that one is a prerequisite for and in need of the other.

The literature review that follows outlines key arguments and debates about interdisciplinarity in general and its positioning in digital research in particular, thus shedding some light on conceptual, intellectual and practical issues that mark interdisciplinarity in digital research. The paper then unpacks the concept of Communities of Practice (CoPs) and suggests that this concept might provide a more interesting perspective on synergistic and collaborative work in digital research compared to the concept of interdisciplinarity. This leads to the presentation of the qualitative methodology of the study and its findings, 
which suggest the development of research communities of practice (RCoPs) in the area of digital research and challenge the popular vision of interdisciplinarity.

\section{Literature review}

\section{Interdisciplinarity}

In the early 1990s, Qiu (1992) found that interdisciplinary research collaboration had steadily increased over a 20-year period. A few years later, Klein and Newell (1998: 393-94) defined interdisciplinarity as 'a process of answering a question, solving a problem or addressing a topic', which 'draws on disciplinary perspectives and integrates their insights through construction of a more comprehensive perspective'.

Almost 20 years later, Szostak (2015) drew on Klein and Newell's definition to outline the stages and practices involved in interdisciplinary research. More specifically, Szostak suggests that interdisciplinarity asks research questions that involve multiple theories and methods of inquiry, and integrates disciplinary-based perspectives in order to achieve a more holistic understanding of the studied phenomena. Thus, one can understand interdisciplinarity as a complex, varying blend of disciplinary thinking and practicing that integrates concepts, techniques and/or data (Porter et al., 2006) and aims to promote a comprehensive and dialectical approach to research knowledge and discovery. In this regard, interdisciplinarity does not signal the dismissal of disciplines; rather, it envisages accommodating multiangle views and cross-thinking that reflect knowledge traditions and practices in more than one discipline, so as to attain a richer understanding of real-world problems.

However, Bammer (2015) has argued that interdisciplinarity appears under certain conditions, and in the following five types of research: first, when research is at the intersection of two disciplines, for instance, biochemistry; second, when research runs across the boundaries of closely related disciplines, such as the disciplines of anthropology and sociology; third, when research draws on a series of disciplinary insights, practices and outputs, such as women's studies; fourth, when research examines the phenomena that run across different disciplines, such as patterning that takes place in various aspects of the natural 
world; and last, in research that involves experts and stakeholders from various disciplines and backgrounds working on a common problem.

Some argue that disciplinary boundaries are shifting, and that interdisciplinarity might provide (much) needed avenues for new research frontiers by combining advantages of two or more disciplines and enhancing the opportunities for scientific breakthroughs (Aram, 2004; Bammer, 2015). Others adopt a more careful approach, arguing that there is a complex relationship between interdisciplinarity and existing disciplines (Barrett, 2012; Bennett, 1997; Klein, 1996). Quite early on, Klein (1996) suggested that disciplinarity and interdisciplinarity are interdependent, since interdisciplinary insights might be assigned varying interpretations in different disciplines. Bennett (1997), in turn, suggested that a discipline is not a natural element of interdisciplinarity, as in the case of women's studies, where research is highly interdisciplinary, but women's studies do not constitute one single discipline. More recently, Bammer (2015) is skeptical of the extent to which practice-based research is truly interdisciplinary ${ }^{\mathrm{i}}$ and argues that the development of research that involves multiple disciplines should lead to appropriate compilation, documentation and transmission of involved concepts and methods, which he calls Integration and implementation Sciences (I2S) $)^{\mathrm{ii}}$ or a new Big-Science-type project (I2S Development Drive) ) $^{\mathrm{iii}}$.

Diverse views also exist in relation to the labelling and naming of research that involves more than one discipline, thus coining different names and terms, such as: multidisciplinarity (Choi and Pak, 2008; Schummer, 2004); transdisciplinarity (Hirsch-Hadorn et al., 2008; Jahn et al., 2012); post-normal science (Funtowica and Ravetz, 1993); sustainability science (Clark, 2007); instrumental interdisciplinarity (Klein, 1996); and epistemological interdisciplinarity (Schummer, 2004) ${ }^{\mathrm{iv}}$. On the basis of 38 scholarly interviews, Lattuca (2001) identified four types of interdisciplinarity: informed disciplinarity, which refers to reaching out to other disciplines or being informed by them; synthetic interdisciplinarity, which indicates a link between disciplines by sharing courses or research questions; transdisciplinarity, which, for Lattuca, takes place when courses and research questions run across disciplines; and conceptual interdisciplinarity, which emerges when intellectual pursuits give space to new intellectual spaces for researchers from different disciplinary backgrounds. 
Among the different terms, multidisciplinarity and transdisciplinarity are the ones used most to describe ways in which we can conduct research that involves various disciplines, while they are often used interchangeably and as equivalent to interdisciplinarity. However, multidisciplinarity and transdisciplinarity are distinct terms and should not be conflated with the concept of interdisciplinarity. Specifically, multidisciplinarity suggests that each discipline identifies and addresses a problem in its conventional method, and then all involved disciplines present their findings side by side (Bammer, 2015).

Transdisciplinarity, on the other hand, suggests that disciplinary researchers conduct research through active cooperation, aiming to generate a shared understanding of and solutions to a research problem, which then guides the research to implement its findings. This means that transdisciplinarity aims at coherence, unity and simplicity of knowledge (Hirsch-Hadorn et al., 2008; Jahn et al., 2012; Szostak, 2015). In this respect, Szostak (2015: 101) describes the relationship between multidisciplinarity, interdisciplinarity and transdisciplinarity as follows: 'Multidisciplinarity can be seen as intermediate between disciplinarity and interdisciplinarity; transdisciplinarity can be seen as interdisciplinarity plus some additional practices'.

Besides labelling, existing theorisations of interdisciplinarity can be considered inadequate, for historical and epistemological reasons. Specifically, the history of interdisciplinary research is longer than the theoretical developments around it, while there is overall a fragmented understanding of interdisciplinarity among scholars (Klein, 1996; Salter and Hearn, 1996) and disciplines (Holmberg and Thelwall, 2014). ${ }^{\mathrm{v}}$ Rather limited work (Frodeman et al., 2010; Klein, 1996; Newell, 1998; Repko, 2012; Szostak, 2015) has contributed theories or methodologies of interdisciplinarity.

In this regard, it has been a challenge for interdisciplinary research to be accepted as mainstream research, while the lack of comprehensive guidance, systematicity and consistency in its practices has made it appear scattered and unorganized. This is supported by Bammer (2013: 3) who has argued that most interdisciplinary research concentrates on the academic margins and 'there is no substantial, wellestablished, internationally accepted methodology'. Bammer (2015) also criticizes the low level of interaction between researchers who conduct interdisciplinary research, something that arguably prevents them from comparing or sharing insights and approaches. This was also shown by Sigelman (2010) who examined how frequently disciplinary terminology moves from one discipline to another and, in a 
comparative study of sociology and political science, he concluded that movement of concepts between the two disciplines is sparse and that interdisciplinary ideas continue to be unlikely to lead to disciplinary outlets.

Hence, interdisciplinarity has generated a series of debates over its practices, mechanisms and outputs, while the term itself covers a range of meanings, often leading to confusion around it. At the same time, interdisciplinarity continues to be seen by many as a double-edged process, in which specializationfragmentation-hybridization come together (Sanz-Menéndez, Bordons and Zulueta, 2001) and comprise a major challenge for individual researchers and research institutions alike.

\section{Interdisciplinarity in digital research: Emerging realities and challenges}

Digital research scholarship has suggested, implicitly or explicitly, the need for collaboration across disciplines and the benefits of interdisciplinarity for digital researchers (Dini et al., 2011; Ess and Dutton, 2013; Holmber and Thelwall, 2014; Hutchinson, 2016; Tsatsou, 2014). As Tsatsou (2014) notes, a range of interdisciplinary research initiatives have made their appearance in digital research over the last more than one decade and one should expect to see more and more diverse and ground-breaking initiatives of this kind in the near future. Ess and Dutton (2013) have specifically referred to Internet studies as a melting pot, one of the ferment examples of the catalyzing interdisciplinary character of ongoing research in this area.

While Internet or digital research and interdisciplinarity are broadly understood as operating hand-inhand, a range of opportunities as well as challenges arise for the researchers involved and the scientific knowledge produced in this area, as argued by Tsatsou (2014: 166):

Internet research often suggests the collaboration of social and computer scientists... This leads to the deployment of new models (e.g., computational social science, agent-based models) and data, the pursuit of large-scale research and the initiation of new practices of collaboration...At the same time, interdisciplinary collaboration in Internet research encounters difficulties in defining interdisciplinary goals, forming explicit interdisciplinary research frameworks, developing mutually shared communication codes and practices, and dealing with varied views on the use of technology and the appropriate use of data. 
An interesting insight into some of these opportunities and challenges was offered by Dini et al. (2011), who studied six digital ecosystem projects in order to construct a theoretical framework of interdisciplinarity in the area. Dini et al. aimed to integrate each project's principles and priorities, and to suggest greater feasibility in integrating disciplines rather than unifying them directly. Dini et al. concluded that interdisciplinary research can derive from radically diverse disciplines that share a goal and collaborate in a broader research community, even if they fail to develop 'a unified theoretical framework due to irreconcilable epistemological differences' (Dini et al., 2011:3).

On the other hand, and similar to the broader approaches to interdisciplinarity, there is a lack of systematic and comprehensive understanding of interdisciplinarity in the digital research domain. This has raised the concern that existing approaches take a rather narrow perspective (Singletary, 2012; Sterne, 2005) that entails a patchy and fragmentary understanding of interdisciplinarity and transdisciplinarity in Internet or digital research. For instance, more than a decade ago, Sterne (2005: 255) acknowledged that digital media studies are heavily reliant on interdisciplinarity and criticized this area of research for not having moved forward as a discipline, and for not constituting a new object of study and research, as disciplines do. Later on, and without the conduct of sufficiently in-depth investigation, Singletary (2012) described a digital future of interdisciplinary knowledge and collaboration, and encouraged online community intelligence as a form of collective discussion and collaboration alongside advancements in digital technology.

More recently, research has shown the existence of disciplinary differences in the research of phenomena which are specific to the digital world. For instance, Holmberg and Thelwall (2014) pointed to disciplinary differences in patterns of research use of microblogging, and pledged for a better understanding of the common and different patterns and drivers of use of Twitter by different disciplines. Similarly, Schroeder and Taylor's (2015) study of patterns of big data research about Wikipedia found that although in some areas researchers from different disciplines build on and extend each other's results, most stay within disciplinary silos. Thus, Schroeder and Taylor argue that researchers must pay more attention to theories and research from other disciplines in order to have a more powerful analytical grasp of the phenomenon they investigate. Some of the latest scholarship also notes that although digital and computational methods are 
increasingly employed by research across a number of disciplines, there is a need to further 'standardize collection and analysis methods of digital media' (Hutchinson, 2016: 2-4).

Overall, there appears to be a call for more interdisciplinary exchange and collaboration, but not much understanding of the internal dynamics of interdisciplinary collaboration in digital research. Thus, according to Tsatsou (2014: 202), the existing approaches to interdisciplinarity in digital research 'essentially signal a multidirectional and partly fragmented effort to methodologically and empirically advance research that involves online media either as research tools and platforms or as actual objects and core elements of research'. While the development and spread of digital technology have arguably enabled research that employs such technology to deliver more fully on its potential (Dini et al., 2011; Goulden et al., 2016; Sterne, 2005; Tsatsou, 2014), there are still questions that need to be answered, such as the questions of: what is the role of the digital in the progression and advancement of interdisciplinarity in all sorts of research work and practices?; and, does digital research unavoidably lead to new and/or more complex forms of interdisciplinarity?

Such questions have not been addressed to date and what we propose in this paper is attendance to the concept of Communities of Practice (CoPs) in relation to digital research. We suggest that the concept of CoPs can help us unpack work in the realm of digital research that involves more than one discipline as a set of practices rather than as a label that lacks concrete or certain meaning and substance, which is what currently happens in the fast evolving and increasingly popular area of digital research. This proposition is precisely what this paper will present from this point onward. However, to do this, we first need to touch upon the notion of CoPs, which is the discussion that follows.

\section{CoPs and their applicability to research}

A volume of scholarly works defines CoPs but definitions vary (Brown and Duguid, 1991; Lave and Wenger, 1991; Wenger, 1998). The concept was first developed by Lave and Wenger in 1991 who concentrated on the idea of informal collaborative learning. Later on, Wenger (1998: 3) defined CoPs as 'groups of people who share a concern or a passion for something they do and learn how to do it better as they interact regularly'. About a decade later, Hara (2009: 3) redefined CoPs and argued that they refer to 
collaborative and informal networks which aim to enhance professional practitioners' shared understandings and engagement in knowledge development.

Scholarly work on CoPs varies and presents different approaches to key concepts in this area, such as the concepts of community, learning, power and conflict, change, formality, and diversity (Cox, 2005: 537). For instance, community was defined by Brown and Duguid (1991) as an informal group that aims to do the same or similar jobs. From a different perspective, Wenger (1998: 3) referred to community as 'a set of social relations and meanings that grow up around a work process when it is appropriated by participants'. Regarding the concept of learning, Lave and Wenger (1991) shed light on informal and situated social interaction rather than on preprogramed processes of cognitive transmission. Brown and Duguid (1991) suggested that learning occurs in a collective way through storytelling, whereas Wenger (1998) stated that an individual learning history involves identification with different practices and trajectories through communities. Regarding power and conflict, Lave and Wenger (1991) demonstrated that power and conflict are constructed in the community between generations and among masters, journeymen and novices. However, Brown and Duguid (1991) criticized these hierarchical relationships and acknowledged that everyone within the community should be the same, while Cox (2005: 530) commented that it is too romantic to think of a community of practice as 'a harmonious collaborative group based on shared meanings'.

CoPs have broadly been studied in the fields of education and learning. In the early phases of elearning, some studies (e.g., Zeichner and Liston, 1996) examined teachers' CoPs and their capacity to resolve teaching problems, implement teaching principles and contribute to teachers' self-development. More recently, research on e-learning in teacher education (e.g., Yandell and Turvey, 2007) suggested that CoPs are useful for making sense of the complexity of teachers' experiences, rather than for narrowing down teachers' role through a standard model (Cochran-Smith, 2004). ${ }^{\text {vi }}$

However, few studies have examined CoPs in the context of scientific research, learning and practices and even these are mostly concerned with specific areas of team-based scientific work and research synergies, such as the application of mixed methods in research (e.g., Denscombe, 2008). An example is that 
of Hemmings et al. (2013) who examined the intragroup social dynamics of a nursing and education research team and found that such dynamics are characterised by processes of socialisation that enable members to cross discipline-bordered traditions and produce interdisciplinary mixed methods combinations. Such mixed methods combinations can be achieved at two levels: the paradigm level, through a shared viewing position and theoretical model; and at the methods and technique levels, through methodological capitalisation and prioritisation and the development of a quantitative culture assessment tool for use in combination with complementary qualitative observation and interview protocols.

Regarding the role of the 'digital', this has been approached in CoP literature, mostly in areas such as e-learning and as an empowering factor for CoPs. Research has argued that one of the most successful ways to reinforce personal knowledge development is the application of CoPs via synchronous discussion platforms (e.g. BBS or MOO), where users investigate their knowledge and exchange their thoughts (Yang, 2009), in accordance with the principle that everyone's contribution is highly valued (Wenger, 1998). From this perspective, digital technology encourages collaborative learning in the community, enriching both individual knowledge construction and community-wide information sharing. This means that the foundations, aims and processes of CoPs which employ digital technology depend on community members' will for continuous change and self-enhancement (Wenger, 1998), as well as on their problem-solving attitudes (Yang, 2009).

However, CoPs have not been considered to date in relation to multi-disciplinary collaborations and team-based work in digital research. The study we present here uses data from ten cases of digital research in five disciplines to demonstrate the need of researchers to develop communities of research sharing and exchange as an application of CoPs, in order to learn from each other's digital research knowledge, experiences and practices. Their views challenge the idea of interdisciplinarity in digital research and plea for research collaboration that crosses disciplines in the form of CoPs, namely in ways that encourage colearning and enhance associated research practices and developments. In what follows, we present the methodology and findings of the study. 


\section{Methodology}

As shown in Figure 1, the study explored the adoption of digital technologies, tools and services by UK-based research in five social science and humanities disciplines: business/management, education, history, literature and politics. The study examined ten cases of UK-based research, two from each of the five disciplines, in order to shed light on researchers' digital research practices and their associated skills and capacity challenges from the stage of designing their research through to data collection and dissemination of research results. In this regard, the study aimed to unpack researchers' voices and explore their views on and experiences of the employment of digital technologies, tools and services in research.

Figure 1 about here

(Having) five disciplines suggests a broad scope of research, with history and literature being located in humanities, management and education belonging to social sciences, and politics being positioned somewhere in between. This scope may be justified by the aim of the study to enhance our understanding of how researchers across social science and humanities employ digital technologies, tools and services, and to uncover related similarities and differences, both within each discipline and across disciplines. Although one of the criteria for selecting the ten cases was the existence of some - even limited - use of digital means of research, it should be noted that unlike other disciplines, such as media and communication and information systems, the selected disciplines are not directly linked to or dependent on the use of digital technologies in research practices. This is important, because it enabled the study to provide a rich picture of the extent to which social science and humanities researchers in the UK keep up with, and take advantage of rapid technological developments in the digital domain. Such an open approach to research cases and disciplines has led us to hypothesise that some of the selected disciplines might be at an early stage of incorporating digital technologies and associated tools and services, while others might show a stronger engagement with digital means of research work.

Before data collection, we reviewed each research case/project. The information needed for the case reviews was found online and by accessing case-specific documentation. Each review involved the collection of background information on the research case in question, and reported on how the case planned 
to use digital technologies, tools and services and whether any research activities in the case had already employed one or more digital means.

Data collection employed a qualitative methodology that consisted of nonparticipant observation (for most research cases) and semi-structured interviews. In the first round of data collection, we collected nonparticipant observation data, with the activities to be observed and the timing and setting of the observations varying according to the case, and being agreed with the leading researchers in each case. However, when observation was not possible or the researcher leading the case did not agree to it, the interactive demonstration of one or two digital-relevant research activities took place to inform the study in a rather 'live' way about activities in which some kind of digital research practice was present. In the second phase, we conducted in-depth semi-structured interviews so as to explore further how digital technologies were used in research and the reasons behind case- and discipline-specific digital research trends and practices. The interview topic guide was designed on the basis of the specifics of each case, the insights gained from the observation or interactive demonstration data of the first round, and the broader aims of the study.

A coding framework was used in the NVivo-assisted analysis of the interview data, which was tested by intercoder reliability testing. NVivo allowed the generation of outputs that complemented the systematic coding and thematic analysis of the interview data, such as text queries, word trees, cluster analysis and reports. NVivo-assisted analysis of the interviews was considered alongside insights gained from nonparticipant observation and interactive demonstration, so that rich qualitative findings were produced. Overall, data analysis was conducted at three levels:

- The case level: analysis of digital research trends, possible contradictions and striking practices in each research case;

- The discipline level: analysis of digital research trends in each discipline, as well as of the similarities and discrepancies between the two cases in the discipline;

- The cross-disciplinary level: analysis of similarities and discrepancies in relation to digital research trends across all five disciplines. 
The next section presents the findings, which shed light on researchers' understanding, views and evaluation of interdisciplinarity in general and their related views and practices in the context of employing digital means in the specific research case in particular.

\section{Findings}

Researchers' general views on interdisciplinarity

Generally, most of the researchers from across the five disciplines demonstrated an open minded way of thinking, without celebrating interdisciplinarity or dismissing it entirely. They related interdisciplinarity to the idea that scholars from different disciplines contribute to a project, while they identified certain areas where methodological and epistemological collaboration is required. Some of the participant researchers were positive as to the idea of interdisciplinary work for the purpose of research impact and the emergence of a new research culture.

Table 1 about here

However, as shown in Table 1, (very) few of the discourses of the participant researchers were concerned with interdisciplinarity, at least in the way this concept is broadly understood. This is not to say that they were not interested in engaging with researchers from other disciplines, but rather, that they did not identify this with the notion of interdisciplinarity. On the contrary, most researchers assigned a more informal and bottom-up character to intellectual and research exchange across disciplines. Thus, most discourses focused on informal research exchange and sharing, which outnumbered those on interdisciplinarity as well as those on discipline-specific principles and practices. The only exceptions were the two cases of history research, where references to disciplinary principles and practices outnumbered those to interdisciplinarity and especially those to informal research exchange and sharing, which did not draw history researchers' attention.

Although the participant researchers' sporadic references to interdisciplinarity were not dismissive, they definitely problematised the vision of interdisciplinarity as a whole. Specifically, some researchers expressed concern about losing sight of the discipline when interdisciplinarity is attempted. They argued that although interdisciplinarity enriches disciplines that engage with it, there is the danger that the discipline is 
not acknowledged sufficiently in the move towards making everybody work together, and for the potential of new synergies. They also highlighted the risk of moving towards interdisciplinary collaboration without sufficiently being acknowledged as a discipline and without adequate understanding of the aims of such collaboration. From this perspective, the PI of the history case at the University of Leeds stated: 'I think there are challenges....it [interdisciplinarity] does enrich research and has to be carefully managed, because otherwise this rush into interdisciplinarily... It's like a box that you tick on a funding application... but why would you be pushing things ahead into interdisciplinary?'

Even those researchers who were concerned with interdisciplinarity somehow more than others, such as the history researchers, problematised its meaning and standing. Specifically, they challenged the rather top-down nature of interdisciplinarity and argued that its agenda is often imposed on researchers by research institutions and funding bodies with the hope that research funding and impact will be attained to the maximum possible degree:

If you take interdisciplinary as it's expressed, which tries to make the boundaries between disciplines more porous...I'm not sure whether that is of use...because that's a process of accelerating change instead of change being organically occurred. It's an imposition on the discipline rather than allowing the discipline to form and develop it by itself (PI, history, University of Leeds).

Interdisciplinarity also seemed to pose questions for researchers regarding their disciplinary identity and the discipline they belong to. For instance, the postdoctoral researcher in the business case at Open University was uncertain whether he would place his work in the field of business research or in that of computer science: 'Because of how the data science grows, I have no idea, so...I mean, it [the area I work in] can be considered computer science or information science... I mean, the kind of online business research I do is down there, in that field.'

\section{Researchers' views on interdisciplinarity at the research case and discipline levels}

Regarding the views of the participant researchers on interdisciplinarity in relation to the research project under study and their discipline as a whole, some interestingly contrasting arguments were put forward. The history researchers who focused on discipline-specific practices and interdisciplinarity more 
than the researchers in the other disciplines provide some interesting examples of such contrasting arguments. On the one hand, the PI of the history case at the UCL emphasised the advantages of the interdisciplinary and digitally-enabled work he does in the specific research project under study:

One of our objectives is to make more of this material [on the history of law] available easily and freely over the Web and advertise that to both disciplines [historians and law researchers] in the hope that they will engage with that [material] more and also in order to create links to scholarship from within these disciplines. ... [we aim to] make the historians better informed of the legal framework of the material they're working on and the legal scholars better informed of the historical context which generated those [legal] documents.

On the other hand, the PI of the history case at the University of Leeds indicated the persistent strength and role of disciplinary identities, as well as the difficulty of blocking discipline-specific labels and practices. Specifically, through reflecting on his identity as a historian, he concluded that disciplinary identity is part of his and other researchers' social identity and mentality:

I'm a historian with a very particular methodology of focus. That's part of my identity as a social being... I think the resistance people detect in the move into interdisciplinarity is because what you're asking them to do is not just a disciplinary shift, but a social shift, because that's part of their social and cultural memory, of who they are.

By using the example of his discipline (i.e. history), this researcher also challenged representations that portray disciplines as static, and suggested the acknowledgement of the historical embeddedness of disciplines and how they have developed over time:

I'm not saying it [his discipline] shouldn't change because things do change, tend to change organically. I mean, history is not the same as a discipline as it was 40 years ago, nor the same as it was in the 19th century, but it is still a discipline and still has an authority, not in a negative sense.

Nevertheless, researchers in other disciplines took a more middle position, such as the PI of the education case at the University of Edinburgh. Although his project was an education project that looked at children's learning and development, it had digital technology at its core, and his closest collaborator was a research assistant with expertise in Informatics. Thus, he talked about opportunities, as well as challenges and difficulties when bringing different disciplinary inputs together. He also brought up questions 
concerning scholarly theoretical work versus technologically focused research and the links of academic research of this kind with consultancy work and external, non-academic actors.

\section{Barriers to interdisciplinarity: a cross-disciplinary perspective}

The participant researchers identified some major challenges in the conduct of interdisciplinary research. Specifically, they considered disciplinary identity/labeling, language, research culture, skills, and time to be the main barriers to interdisciplinary work, while they highlighted funding as the stimuli for such work to be carried out.

Figure 2 about here

As shown in Figure 2, barriers to interdisciplinarity were raised in different research cases across different disciplines. However, time (i.e. time constraints) was presented as the most important barrier and was mentioned in almost all studied research cases (9 out of 10) and in all five disciplines. Thus, when, for instance, the PI of the history case at the University of Leeds was asked whether he had considered collaborating with experts from other disciplines such as computer scientists, he stated: 'it was just the constraints of time and money that didn't allow us to do that but the next research project is going to be interdisciplinary.' The next most important barrier appeared to be that of skills, which indicated the existence of some gaps in the researchers' literacy, capacity and/or knowledge required for collaborating with researchers from other disciplines. For instance, the PI of the business case at the University of Manchester illustrated that although there existed a highly collaborative environment in their institution, there was a skills issue and he stressed the need for big data training.

Other barriers to interdisciplinarity for the participant researchers were disciplinary factors, such as those of discipline-specific research culture and disciplinary identity/labelling. Six cases across four disciplines (except for business research) brought up the constraint of research culture, and researchers in four cases across four disciplines (except for politics) stressed the role of disciplinary identity and labelling. For instance, the researchers in the two education cases highlighted the rather closed and resistant attitude to interdisciplinarity in their field, especially in relation to the conduct of digitally-enabled research. Also, the PI of the history case at the University of Leeds pointed out difficulties in integrating different research 
cultures and compared historians' with social scientists' methodologies: 'as a historian, I would interrogate an archival source to a much greater extent than just interviewing that source and getting the transcribing of what people say and whether what they say actually means something'.

Language was the least mentioned barrier, with only two cases in humanities research making reference to it. Specifically, the PI of the literature case at the University of Leicester talked about the difficulty finding an all-round researcher for a project like his (i.e. a digital humanities project) 'who could do all that stuff [the digital humanities side of the work] and negotiate with OUP [Oxford University Press] and everybody else and talk their language'.

On the other hand, funding was mentioned as the impetus of interdisciplinary research in five cases from across three disciplines (except for business and politics research). For instance, the PI of the literature case at the University of Leicester pointed out that the existing external funding mechanisms comprise the major force for humanities researchers to put together collaborative interdisciplinary bids and demonstrate their competence in digital humanities. Specifically, he stated that the increase of funding from the Arts and Humanities Research Council over the last few years has come at a price, as the Council requires you (i.e. the researcher) to 'present your project through a science-like model of team work', which 'is quite difficult to do'.

\section{The alternative of $R C o P s$}

As shown in Table 1 above, while some of the participant researchers focused on disciplinary discourses, most engaged with the ideas of informal research sharing and exchange as a means to enhance their skills and knowledge in general, and for employing digital means of research in particular. They favoured the development of Research Communities of Practice (RCoPs) that would enhance the employment of digital means in scientific research on the grounds of informal collaboration, experience exchange and knowledge sharing across different disciplines.

As shown in Figure 3, the alternative of RCoPs was put forward at the case, discipline and crossdisciplinary levels. Specifically, there were three main perspectives that the participant researchers adopted to portray their vision of RCoPs: 'knowledge sharing', 'experience exchange' and 'informal collaboration'. 
The researchers valued the exchange of research experiences and the sharing of knowledge, especially in relation to the use of digital technologies for research dissemination and knowledge exchange purposes.

Figure 3 about here

More specifically, a desire for sharing knowledge and related resources was expressed in all ten research cases studied. The politics researchers at the University of Nottingham, for instance, expressed their desire to get together with other researchers to share ideas and thoughts. Also, the PI of the literature case at the University of Leicester demonstrated the connection of 'digital humanities' with the idea of sharing information, while the PI of the history case at the University of Leeds stressed the importance of 'sharing the research with both other researchers and members of the general public and students'. Researchers in four studied cases adopted the idea of informal collaboration. The PI of the literature case at the University of Glasgow, for instance, stated about the Burns project:

one of the things I'd love to do in the future is revisiting the [interactive, online] maps and speaking to Visit Scotland, speaking to other organizations and ask them "Are you aware of this? ... is there a way to use this, to disseminate it? Are there any ways in which you might want to collaborate and develop it with us?"

Similarly, five cases and mostly education research stressed the importance of exchange of experiences. The words of the PI of the education case at the University of Edinburgh are indicative in this respect:

if someone says "I went on LinkedIn, ta ta ta"...through in-person communication you get a feeling of how much one has been helped by it... I want someone to give me an overview of what is out there, what I'm not doing it, what are people's experiences... and it's difficult sometimes to capture people's experiences in the training.

At the same time, the participant researchers argued that research institutions and universities could and should facilitate such forms of knowledge sharing, experience exchange and informal collaboration across disciplines by taking initiatives and encouraging researchers to come together, open up and develop a dialogue. This challenges institutions' top-down, prescribed and mostly utilitarian agendas for the development of interdisciplinary research, and invites the development of new agendas that will openly and 
responsively aim at bottom-up, organically grown and flexible forms of informal collaboration, knowledge sharing and experience exchange across disciplines, including the field of digital research.

\section{Conclusion}

In studying digital research practices in ten research cases from five disciplines in the humanities and social sciences in the UK, we reached some interesting conclusions about interdisciplinarity, and more specifically, researchers' experiences with and ambitions for synergies and collaboration with different disciplines.

The study found that few of the participant researchers were concerned with interdisciplinarity, in the way that this concept is presented in scholarship and formal discussions. However, this does not to mean that they were not interested in or concerned with pursuing collaborative research with other disciplines. It is, rather, that their vision of such collaborative research did not identify with how the notion of interdisciplinarity is broadly understood. Even those concerned with interdisciplinarity problematised its meaning and standing. Specifically, they challenged the rather top-down nature of interdisciplinary research, and argued that interdisciplinarity is often imposed with the aim of attaining research funding and maximising research impact.

Further, the study found that humanities and social science researchers in the UK favour bottom-up knowledge sharing and experience exchange with other researchers and informal research collaboration across disciplines. They also argued that research institutions and universities should facilitate informal collaboration, knowledge sharing and experience exchange in the research community by supporting and encouraging bottom-up initiatives that bring researchers from different disciplines together and make them open up and develop a dialogue with each other. In this regard, they confidently supported the alternative of RCoPs, challenging the popular vision of interdisciplinarity in general and in the realm of digital research in particular.

Through exploring researchers' views and experiences of interdisciplinarity in the domain of digital research, this study makes a two-fold contribution. First, it provides an insight into researchers' take on interdisciplinarity from the perspective of digital research, since the existing literature lacks an overarching 
understanding of the mechanics and dynamics of interdisciplinarity and overlooks researchers' own understanding and experiencing of it. Second, the paper sheds light on the potential of developing RCoPs as a route for taking up the challenge of digital means of research and other research developments that run across disciplines, thus providing an alternative to the popular mandate of interdisciplinarity and filling in a gap in the literature. Hence, the study argues for a new agenda; an agenda that promotes the development of RCoPs which are driven by researchers themselves, but are actively supported by research institutions and Universities too.

However, this study has a number of limitations. Although its scope spanned five disciplines, its sample size was moderate, and more than two research cases per discipline would most likely offer richer insights. Also, data collection methods and techniques varied for some of the cases studied, with participant observation being applied to most, but not all cases. Although this was a necessity for practical reasons, it raises questions with regard to the comparability of the collected data and the insights gained for the cases where participant observation was not conducted.

Nevertheless, this study provides a useful starting point in order to dig deeper into the challenges of and alternatives to interdisciplinarity in research fields where cross-disciplinary collaboration is taken for granted, such as that of digital research. It also comprises a study that offers lessons for institutional and funding bodies which appear to consider interdisciplinarity the only possibility for collaborative research between two or more disciplines, inviting them to consider research collaboration undertakings jointly with researchers and in the direction of developing the alternative of RCoPs.

\section{Notes}

${ }^{\mathrm{i}}$ According to Bammer (2015: 4), research groups and teams that work on practice-based advances tend to be isolated from each other.

ii Bammer (2015: 5-6) argues that there is no best way to conduct research when diverse disciplines are involved and suggests a new discipline - Integration and Implementation Sciences (I2S) - that will 
document and transmit the concepts and methods that underpin all different options and styles in conducting interdisciplinary research.

iii For Bammer, compiling and documenting concepts and methods that involve multiple disciplines will take decades. So he suggests to accelerate the process through a new 'Big Science'-type project, which he calls I2S Development Drive (2015: 12).

${ }^{\text {iv }}$ Klein (1996) argued that while instrumental interdisciplinarity involves bridging different fields, epistemological interdisciplinarity focuses on restructuring a former approach to defining a field.

${ }^{v}$ Holmberg and Thelwall (2014) argued about the impossibility of a unified theoretical framework for interdisciplinarity due to irreconcilable epistemological differences among disciplines.

vi The standard model - also called a 'competences approach' - has become increasingly important, but Cochran-Smith (2004: 208) calls for a space where open critique of the outcomes of teacher education can take place.

\section{References}

Aram, J.D. (2004). Concepts of interdisciplinarity: Configurations of knowledge and action. Human Relations, 57(4), 379-412.

Bammer, G. (2013). Disciplining Interdisciplinarity: Integration and Implementation Sciences for Researching Complex Real-World Problems. Canberra: ANU E Press.

Bammer, G. (2015). Interdisciplinarity: less vague please. Nature: International Weekly Journal of Science 526(7574): 506. doi: 10.1038/526506c.

Barrett, B.D. (2012). Is interdisciplinarity old news? A disciplined consideration of interdisciplinarity. British Journal of Sociology of Education, 33(1), 97-114. doi: 10.1080/01425692.2012.632868. Bennett, T. (1997). Culture: A Reformer's Science. Thousand Oaks, CA: Sage.

Brown, J.S. and Duguid, P. (1991). Organizational learning and communities of practice: toward a unified view of working, learning and innovation. Organization Science, 2(1), 40-57. 
Choi, B.C. and Pak, A.W.P. (2008). Multidisciplinarity, interdisciplinarity, and transdisciplinarity in health research, services, education and policy: 3. Discipline, inter-discipline distance, and selection of discipline. Clinical \& Investigative Medicine, 31(1), 41-8.

Cochran-Smith, M. (2004). Defining the outcomes of teacher education: What's social justice got to do with it? Asia-Pacific Journal of Teacher Education, 32(3), 193-212.

Clark, W.C. (2007). Sustainability science: a room of its own. Proceedings of the National Academy of Sciences of the United States of America, 104(6), 1737-8.

Cox, A. (2005). What are communities of practice? A comparative review of four seminal works. Journal of Information Science, 31(6), 527-40.

Dini, P., Iqani, M. and Mansell, R. (2011). The (Im)possibility of interdisciplinarity: Lessons from constructing a theoretical framework for digital ecosystems. Culture, Theory and Critique, 52(1), 327.

Ess, C. and Dutton, W.H. (2013). Internet studies: perspectives on a rapidly developing field. New Media \& Society, 15(5), 633-43.

Frodeman, R., Klein, J.T. and Mitcham, C. (eds.) (2010). The Oxford Handbook of Interdisciplinarity. New York: Oxford University Press.

Funtowica, S.O. and Ravetz, J.R. (1993). Science for the post-normal age. Futures, 25(7), 739-55.

Goulden, M., Greiffenhagen, C., Crowcroft, J et al. (2016). Wild interdisciplinarity: Ethnography and computer science. International Journal of Social Research Methodology, 20(2), 137-50. doi: 10.1080/13645579.2016.1152022.

Hara, N. (2009). Communities of Practice: Fostering Peer-to-Peer Learning and Informal Knowledge Sharing in the Work Place (Vol. 13). Springer Science \& Business Media.

Hirsch-Hadorn, G., Biber-Klemm, S., Grossenbacher-Mansuy, W et al. (2008). The emergence of transdisciplinarity as a form of research. In Hirsch-Hadorn G, Hoffmann-Riem SH, Biber-Klemm et al. (Eds.) Handbook of Transdisciplinary Research. Springer: Dordrecht, pp. 19-39. 
Hemmings, A., Beckett, G., Kennerly, S et al. (2013). Building a community of research practice:

Intragroup team social dynamics in interdisciplinary mixed methods. Journal of Mixed Methods Research, 7(3), 261-73.

Holmberg, K. and Thelwall, M. (2014). Disciplinary differences in Twitter scholarly communication. Scientometrics, 101(2), 1027-42. doi: 10.1007/s11192-014-1229-3

Hutchinson, J. (2016). An introduction to digital media research methods: how to research and the implications of new media data. Communication Research and Practice, 2(1), 1-6, doi:10.1080/22041451.2016.1155307.

Jahn, T., Bergmann, M. and Keil, F. (2012). Transdisciplinarity: Between mainstreaming and marginalization. Ecological Economics, 79, 1-10.

Klein, J.T. (1996). Crossing Boundaries: Knowledge, Disciplinarities, and Interdisciplinarities. Charlottsville, London: University Press of Virginia.

Klein, J.T. and Newell, W.H. (1998). Advancing interdisciplinary studies. In W.H. Newell (Ed.) Interdisciplinarity: Essays from the Literature. New York: The College Board, pp. 3-22.

Lave, J. and Wenger, E. (1991). Situated Learning: Legitimate Peripheral Participation. Cambridge: Cambridge University Press.

Lattuca, L.R. (2001). Creating Interdisciplinarity. Nashville, Tennessee: Vanderbilt University. Mills, D. and Ratcliffe, R. (2012). After method? Ethnography in the knowledge economy. Qualitative Research, 12(2), 147-64.

Newell, W.H. (1998). Professionalizing interdisciplinarity: Literature review and research agenda. In Newell WH (Ed.) Interdisciplinarity: Essays from the Literature. New York: The College Board, pp. 529-63. Pohoryles, R.J. and Alice, B.M.V. (2011). Interdisciplinarity as a challenge. The European Journal of Social Science Research, 24(4), 391-3.

Porter, A.L., Roessner, J.D., Cohen, A.S et al. (2006). Interdisciplinary research: meaning, metrics and nurture. Research Evaluation, 15(3), 187-95. doi: 10.3152/147154406781775841.

Qiu, L. (1992). A study of interdisciplinary research collaboration. Research Evaluation, 2(3), 169-75. doi: 10.1093/rev/2.3.169. 
Repko, A.F. (2012). Interdisciplinary Research: Process and Theory. London: Sage.

Salter L and Hearn A (1996) Outside the Lines: Issues in Interdisciplinary Research. London: McGillQueens's University Press.

Sanz-Menéndez, L., Bordons, M. and Zulueta, M.A. (2001). Interdisciplinarity as a multidimensional concept: its measure in three different research areas. Research Evaluation, 10(1), 47-58. doi: $10.3152 / 147154401781777123$.

Schroeder, R. and Taylor, L. (2015). Big data and Wikipedia research: social science knowledge across disciplinary divides. Information, Communication \& Society, 18(9), 1039-56, doi:

$$
\text { 10.1080/1369118X.2015.1008538 }
$$

Schummer, J. (2004). Multidisciplinarity, interdisciplinarity, and patterns of research collaboration in nanoscience and nanotechnology. Scientometrics, 59(1), 425-65. doi:10.1023/B:SCIE.0000018542.71314.38

Sigelman, L. (2010). Terminological interchange between sociology and political science. Social Science Quarterly, 91(4), 883-905, doi:10.1111/j.1540-6237.2010.00740.x

Sterne, J. (2005). Digital media and disciplinarity. The Information Society, 21(4), 249-56.

Singletary, K. (2012). Interdisciplinary intellect: HASTAC and the commitment to encourage collective intelligence. Arts and Humanities in Higher Education, 11(1), 1-16.

Szostak, R. (2015). Extensional definition of Interdisciplinarity. Interdisciplinary Studies, 33, 94-116.

Tsatsou P (2014). Internet Studies: Past, Present and Future Directions. Surrey: Ashgate Publishing.

Tsatsou P (2016). Can media and communication researchers turn the present challenges of research impact and interdisciplinarity into future opportunities? The International Communication Gazette 78(7): 650-6. doi: 10.1177/1748048516655718.

Wenger, E. (1998). Communities of Practice: Learning, Meaning and Identity. Cambridge: Cambridge University Press.

Yandell, J. and Turvey, A. (2007). Standards or communities of practice? Competing models of workplace learning and development. British Educational Research Journal, 33(4), 533-50. 
Yang, S.H. (2009). Using blogs to enhance critical reflection and community of practice. Educational

Technology \& Society, 12(2), 11-21.

Zeichner, K. and Liston, D. (1996). Reflective Teaching: An Introduction. Mahwah, NJ: Lawrence Erlbaum.

Figure 1: Study framework

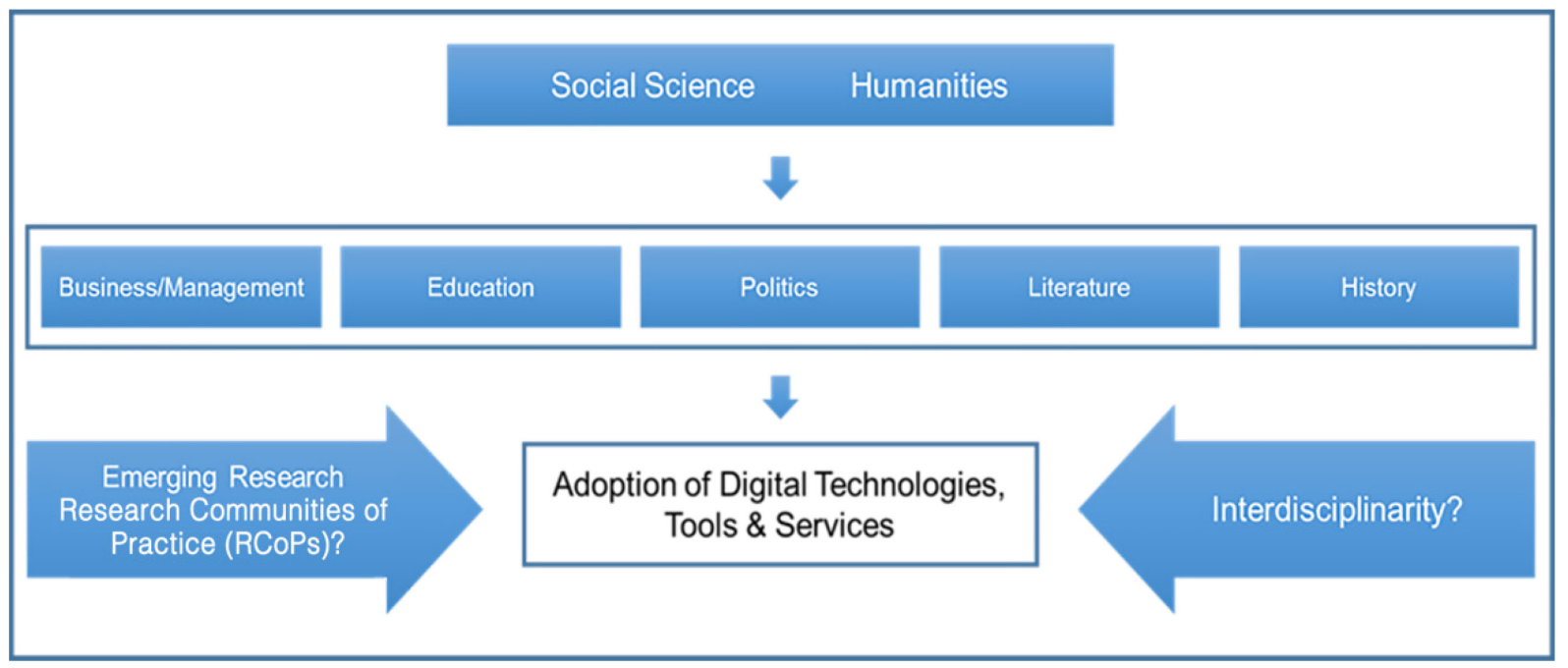

Figure 2: Barriers to interdisciplinarity

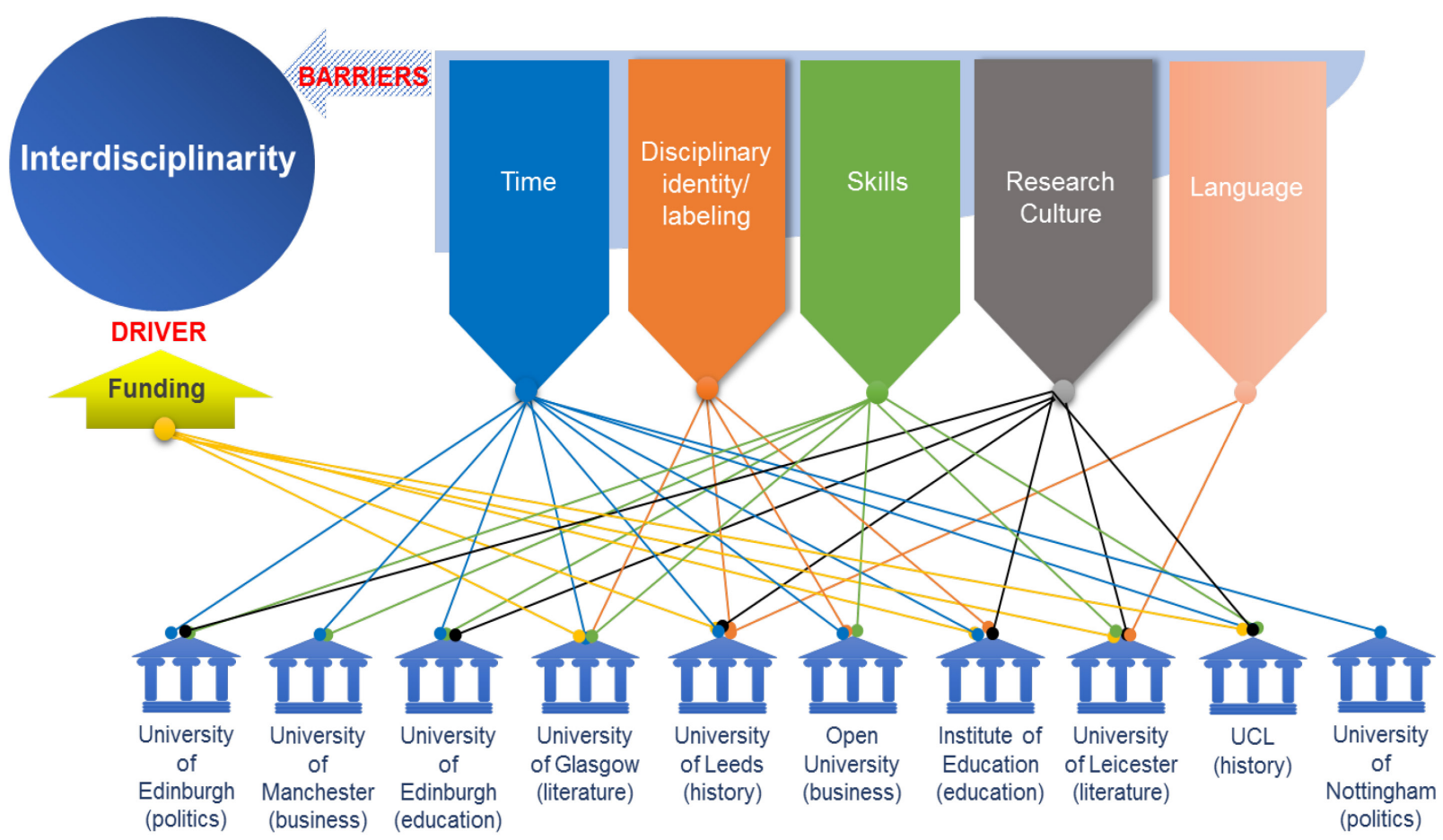


Figure 3: The alternative of Research Communities of Practice (RCoPs)

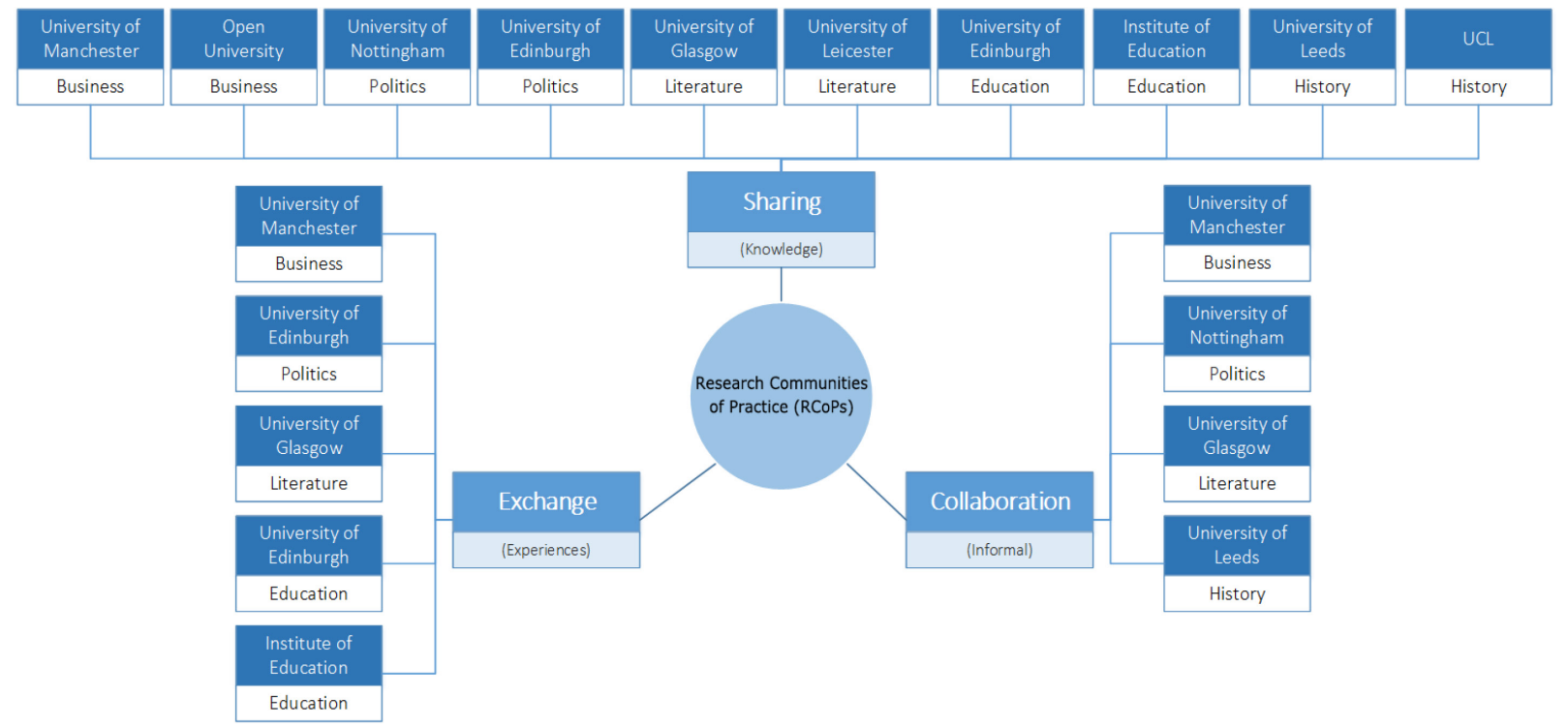

Table 1: Focus of participant researchers' interview discourses

\begin{tabular}{|c|c|c|c|c|c|}
\hline \multirow{2}{*}{ Focus of discourse } & \multicolumn{5}{|c|}{ Discipline } \\
\hline & Business & Education & History & Literature & Politics \\
\hline Disciplinary principles \& practices & 6 & 4 & 37 & 1 & 1 \\
\hline Interdisciplinarity & 2 & 0 & 15 & 0 & 3 \\
\hline Informal research exchange and sharing & 18 & 26 & 4 & 14 & 28 \\
\hline
\end{tabular}


Yupei Zhao (PhD University of Leicester) is a Senior Research Fellow in the School of Communication and Design at the Sun Yat-Sen University of China. Her research interests include new media and civil society, political communication, Internet governance, citizen mobilization, and social media.

Panayiota Tsatsou, is an Associate Professor of Media and Communication at the University of Leicester. Her research interests lie in the broader field of digital media and her publications aim at intellectual and research advancement in the following areas: digital divides/digital inclusion, Internet studies, digital research, digital media and civic participation and digital policy and regulation. More info on her work can be found at http://www2.le.ac.uk/departments/media/people/dr-panayiota-tsatsou. 